\title{
A NOTE ON INFINITESIMAL HOLONOMY GROUPS*
}

\author{
ALBERT NIJENHUIS
}

In a recent paper [1], H. Ozeki has extended the author's results [2] on local and infinitesimal holonomy groups for connections in linear fiber bundles (whose fiber is a vector space) to general fiber bundles whose structure group is a Lie group. Ozeki's Lemma 7 (corresponding to the author's Lemma 5.2) appears to be rather crucial in the development, while the proof is somewhat involved.-This note intends to present a more direct proof of the lemma, stating a case in which the local holonomy group $H^{*}(x)$ and the infinitesimal holonomy group $H^{\prime}(x)$ coincide:

If $\operatorname{dim} H^{\prime}(x)$ is constant in a neighborhood of $x$ in $P$, then we have $H^{\prime}(x)$ $=H^{*}(x)$.

In the proof we use Ozeki's notations and terminology.-There is a neighborhood $U^{\prime}$ of $p=\pi \cdot x$ in $M$ such that $\operatorname{dim} \mathfrak{h}^{\prime}(y)$ is constant for all $y \in U$ $=\pi^{-1}\left(U^{\prime}\right)$. At every point $y \in U$ there is therefore a vertical plane $W_{y}$ in the tangent space, of dimension $h=\operatorname{dim} \mathfrak{h}^{\prime}(y)$ consisting of the values at $y$ of those vertical vector fields over $P$ that represent infinitesimal right transformations on $P$ by elements of $\mathfrak{h}^{\prime}(y)$. The distribution $W$ of $h$-dimensional planes over $U$, thus arising, is in involution (i.e., the Lie bracket of any two vector fields belonging to the distribution, also belongs to it), as can be shown to follow from the fact that $\mathfrak{h}^{\prime}(y)$ is a Lie algebra, and that $\mathfrak{h}^{\prime}(y)$ is of type ad (G) (cf. [1] Prop. 2 ii). ${ }^{1)}$ The distribution $H$ of horizontal planes is not in involution, but the bracket of any two vector fields of $H$ can be decomposed into a vector fields of $H$ and one of $W$. In fact, if the two vector fields are horizontal liftings

\footnotetext{
Received June 4, 1957.

* Research sponsored by the Office of Naval Research.

1) Another way of seeing this is by remarking that if $X, Y$ are right-invariant horizontal vector fields of $P$, then over each fiber the vertical part $v[X, Y]$ of the Lie bracket $[X, Y]$ belongs to $W$, and represents an infinitesimal admissible map of each fiber into itself. More generally, the vertical vector fields $\left[V_{h}, \ldots,\left[V_{1}, v[\mathrm{X}, Y]\right] \ldots\right]$ represent infinitesimal admissible maps, span $W$, and correspond exactly to the generators [1] $\left(I_{h}\right)$ of $l^{\prime}(x)$ by $2 V_{k} \ldots V_{1}, \ldots(X, Y)=-\omega\left(\left[V_{k}, \ldots,\left[V_{1}, v[X, Y]\right] \ldots\right]\right)$.
} 
of vector fields in the base space whose bracket there vanishes, then the bracket of the lifted vector fields belongs to $W$. This follows from the fact that $\mathfrak{h}^{\prime}(x)$ contains the values of the curvature form. Finally, the bracket of a vector field in $H$ and one in $W$ decomposes into the sum of an element of $H$, and one of $W$; in particular, if the horizontal one and the vertical one are invariant under right-transformations of $P$, then the bracket is right-invariant, and belongs to $W$. This is a consequence of the construction of $\mathfrak{h}^{\prime}(x)$ as spanned by the values of the curvature form and derivatives. Hence the distribution $H+W$ is in involution, and by Frobenius' theorem there is an integral manifold passing through $x$, projecting down on a neighborhood $U^{\prime \prime}$ of $p=\pi \cdot x$. All horizontal curves starting from $x$, whose projection on $M$ is inside $U^{\prime \prime}$, must lie inside the integral manifold, of dimension $\operatorname{dim} M+h$. Hence this manifold contains $P\left(U^{\prime \prime}, x\right)$. Thus we find $\operatorname{dim} H^{*}(x) \leqq \operatorname{dim} H^{\circ}\left(U^{\prime \prime}, x\right)=\operatorname{dim} P\left(U^{\prime \prime}, x\right)-\operatorname{dim} M$ $\leqq(\operatorname{dim} M+h)-\operatorname{dim} M=h=\operatorname{dim} H^{\prime}(x)$. Proposition 2 (i) of [1] states $\operatorname{dim} H^{\prime}(x) \leqq \operatorname{dim} H^{*}(x)$, and from these two facts one finds the desired equality $H^{\prime}(x)=H^{*}(x)$.

\section{LITERATURE}

[1] H. Ozeki. Infinitesimal holonomy groups of hundle connections. Nagoya Math. J., 10, 105-123 (1956).

[2] A. Nijenhuis. On the holonomy groups of linear connections. Kon. Nederl. Akad. Wetenschappen Amsterdam, Proc., A 56 (3), 233-249, A 57 (1), 17-25 (=Indag. Math., 15 (3), 233-249; 16 (1), 17-25).

University of Washington

Seattle, Washington, U.S.A. 